\title{
Das westlykische Daidala in der Kaiserzeit: Eine neue Inschrift und historische Überlegungen
}

\author{
Werner TIETZ*
}

\section{Vorlage einer neuen Inschrift aus İnlice, dem antiken Daidala}

Die hier vorzustellende Inschrift befindet sich heute in Göcek (Türkei, Provinz Muğla) im antiken lykisch-karischen Grenzgebiet. Entdeckt wurde sie in der nahe gelegenen Streusiedlung İnlice, etwa $20 \mathrm{~km}$ westlich von Fethiye und $7 \mathrm{~km}$ östlich von Göcek. Der Text steht auf einem wohl ehemals quaderförmigen später - vermutlich für die Nutzung in einer Ölmühle - zylindrisch zugerichteten Block aus lokalem, gelblichem Kalkstein. Der Block ist zu stark, um zu einer Stele oder einem Sarkophag zu gehören, und so liegt es nahe, dass er ursprünglich in ein Grabhaus oder ein anderes größeres Grabmonument verbaut war.

Die Fundumstände sind dadurch verunklärt, dass der Stein 1999 vom Fundort nach Göcek verbracht wurde, um dort einen Garten zu zieren. Am selben Tag noch wurde der Stein vom Autor photographiert (s. Abb. 1) und die erhaltenen Textteile abgeschrieben. Eine eingehende Untersuchung, Vermessung oder auch die Anfertigung eines Abklatsches war damals aus rechtlichen Gründen nicht möglich, und großer Dank gebührt nun Fatih Onur (Antalya), der nach so langer Zeit die erstmalige Publikation des Textes auf Grundlage der damals gemachten Abschrift und Photographien ermöglicht hat ${ }^{1}$.

Seit der Entdeckung der Inschrift hat Göcek sein Gesicht stark verändert, und aus einem noch 1999 eher verschlafenen Ort mit bescheidenem Yachthafen ist im Boom von Wirtschaft und insbesondere Tourismus in den frühen 2000er Jahren eine weit ausgedehnte Kleinstadt mit zahlreichen neuen Wohn- und Geschäftsvierteln geworden. Aufgrunddessen konnte die Inschrift seitdem nicht mehr aufgefunden und kein Abklatsch hergestellt werden. Der damalige ortskundige Tippgeber des Autors ist leider schon vor mehr als zehn Jahren verstorben. Die sehr gut erhaltenen, sauber und regelmäßig gearbeiteten Buchstaben lassen freilich kaum einen Zweifel an ihrer Lesung, so dass von einem Abklatsch nur wenig weiterer Aufschluss zu erwarten wäre.

\section{Die Buchstaben und ihre Datierung:}

Die Inschrift ist als annähernd rechteckige Kolumne gestaltet, und die regelmäßigen Buchstaben etwa $7 \mathrm{~cm}$ groß, mit annähernd gleichem Abstand voneinander. Sie sind mit Apices ausgestattet. Die Schenkel von My und Ny sind senkrecht ausgeführt, die des Sigma waagrecht. Das Pi zeigt einen an den beiden gleich langen, senkrechten Hasten abschließenden Querbalken. Das Theta weist im Kreis eine kurze Querhaste auf, was in Kaunos erstmals in augusteischer Zeit belegt ist. Das Xi besteht aus drei unverbundenen, waagrechten Hasten. Das Alpha zeigt eine gebrochene

\footnotetext{
* Prof. Dr. Werner Tietz, Universität zu Köln, Historisches Institut, Alte Geschichte, Albertus-MagnusPlatz, D 50923 Köln, Deutschland (werner.tietz@uni-koeln.de; https://orcid.org/0000-0002-8280-8905).

${ }^{1}$ Dank gebührt ihm und Christof Schuler (München) auch für einige wertvolle Hinweise.
} 
Mittelhaste. Das große Omega ist unten nahezu geschlossen und besitzt lange, horizontale Hasten mit nach oben gezogenen Enden.

Aus İnlice selbst oder der näheren Umgebung ist bislang lediglich die sehr kurze Weihinschrift eines rhodischen Epistates an Tyche und Aphrodite erhalten, die ohne jeden Zweifel aus der Zeit stammt, als der Platz zusammen mit großen Teilen der lykischen Küste Teil der integrierten rhodischen Peraia war und sich vermutlich nahe dem 'Dorischen Felsgrab' an der heutigen Landstraße fand ${ }^{2}$. Sie ist wesentlich

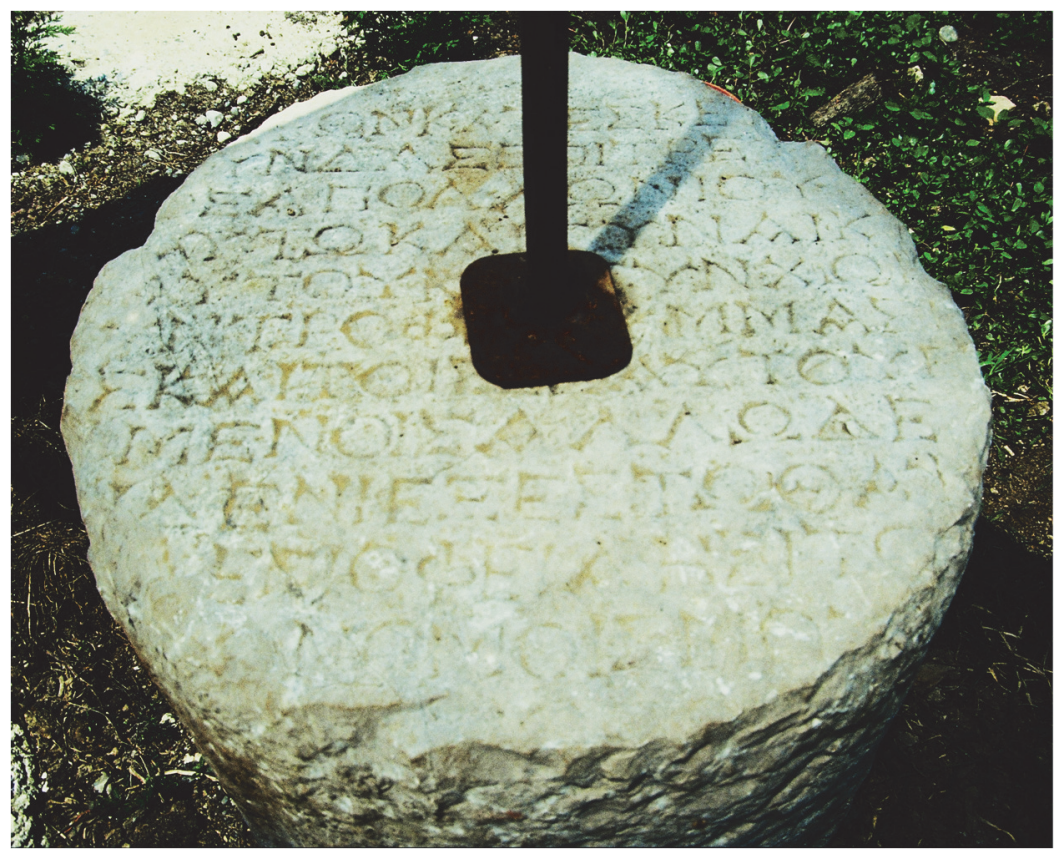

Abb. 1) Inschrift aus Inlice, heute in Göcek (Photo W. Tietz 1999) älter als der hier vorzustellende Text, da die Kreise bzw. Ellipsen in der Weihinschrift des Epistates recht klein ausgeführt sind, die Mittelhaste des Alpha gerade ist und die rechte Haste des Pi kürzer als die linke ist. Im Kontext der Epigraphik der weiteren Umgebung sind My, Ny und Sigma zwischen dem 3. Jh. v.Chr. und dem 1. Jh. n.Chr. anzusetzen, das Pi ab dem 1. Jh. v.Chr., das Xi in die Kaiserzeit, das Alpha und das Omega in die Zeit ab dem 1. Jh. v.Chr., wobei Chr. Marek, auf dessen Analyse des kaunischen Corpus sich diese Datierungen einzelner vor allem stützen, darauf hingewiesen hat, dass dort durchaus ältere Buchstabenformen in jüngeren Inschriften auftauchen, Datierungsversuche auf Grundlage paläographischer Kriterien mithin riskant sind ${ }^{3}$. Paläographisch vergleichbar sind ferner aus Telmessos die klar hellenistischen Inschriften TAM II 71 sowie TAM II 92 (nach dortigen Faksimiles), jedoch weist jeweils das Alpha dort eine ungebrochene Mittelhaste auf. Negativ fallen hingegen Vergleiche mit anderen nahen Fundplätzen aus, insbesondere mit dem kaiserzeitlichen Corpus aus Lydai ${ }^{4}$ und mit den in TAM publizierten hellenistischen Inschriften aus Lissai ${ }^{5}$. Im Vergleich zu einer weiteren späthellenistischen (1. Jh.

2 TAM II 163. Zur Datierung ins 2. Jh. v.Chr. anhand paläographischer Kriterien s. ebenda. Fraser Bean 1954, 87 datieren den Text vorsichtig "to the first century B.C., or possibly to the Imperial period". Die Herkunft dieses Steins ist umstritten. Vermutet wurden bislang sowohl İnlice als auch die vorgelagerte Insel Tersane Adas1, wo er gefunden wurde: Fraser - Bean 1954, 55; Roos 1969, 92; Tietz 2003, 237-238. Eine weitere Inschrift befindet sich auf einem wohl kaiserzeitlichen Altar im heutigen Dorf İnlice auf einem Altar. Sowohl die sehr schlechte Erhaltung als auch die schwierige Fundlage haben eine Lesung oder gar Publikation bislang verhindert: Ebenda 236.

\footnotetext{
${ }^{3}$ Marek 2006, 111-116.

${ }^{4}$ TAM II 129-157.

5 TAM II 158-161.
} 
v.Chr./1. Jh. n.Chr.) Inschrift aus Lissai fällt auf, dass diese aufgrund des rechtwinklingen Sigma jünger erscheint, ansonsten aber paläographisch der hier präsentierten Inschrift ähnlich ist ${ }^{6}$.

Zusammenfassend deuten die paläographischen Eigenschaften des Textes im Vergleich mit dem kaunischen Corpus auf eine Datierung in das 1. Jh. v.Chr. oder das 1. Jh. n.Chr., mit einem leichten Schwerpunkt auf dem 1. Jh. n.Chr.

Photo: W. Tietz 1999.

Datierung: 1. Jh. v.Chr./1. Jh. n.Chr.

vacat

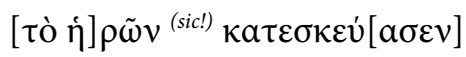

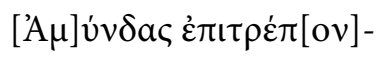

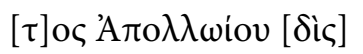

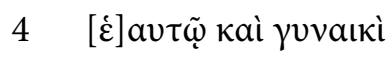

[غ่] $\alpha v \tau \tau \tilde{v}, \kappa[\alpha i]] \sigma v v \chi \omega \rho[\tilde{\omega}]$

$[\sigma] v v \tau \rho o ́ \varphi[\omega ⿻ 上 丨] v \mu \mu \alpha \sigma[\mathrm{l}]$

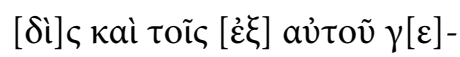



$[\mu] \eta ̣ \delta \varepsilon v \grave{\imath} \varepsilon \xi \xi \dot{\varepsilon} \sigma \tau \omega \theta \dot{\alpha} \psi[\alpha \mathrm{l}]$

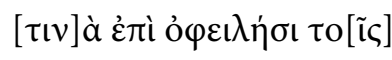



Übersetzung:

„[Das Grab] hat gebaut Amyndas mit Erlaubnis des Apollonios, [Sohn des Apollonios], für sich und seine Frau, und ich erlaube (die Bestattung) auch meinem Ziehbruder Symmasis, Sohn des Symmasis und dessen Nachkommen. Einem anderen aber ist es nicht erlaubt, jemanden (hier) zu bestatten, sonst soll er meinen Erben - - schulden."

vacat

\section{Ergänzung der verlorenen Textteile:}

Der Inschriftenträger wurde dem Anschein nach für landwirtschaftliche Zwecke von einem Quader in eine Walze umgearbeitet, nach dem heutigem Zustand zu urteilen, um damit eine Ölmühle herzustellen. Dies erfolgte vor der Verbringung von İnlice nach Göcek, aufgrund der verwendeten eisernen Materialien aber in rezenter Zeit. Ob der Stein bis dahin als Quader erhalten geblieben oder wie lange er als Mahlstein in Verwendung war, lässt sich nicht sagen. Die erhaltenenen Buchstaben und Buchstabenreste sind nahezu alle sehr gut lesbar.

Trotz einiger Gebrauchsspuren ist am oberen und unteren Rand des Textes deutlich zu erkennen, dass dort mehr freier Raum vorhanden ist als zwischen jeder der Zeilen. Es liegt also nahe, dass keine Zeile ganz verloren ist. Die Möglichkeit einer etwa durch größere Buchstaben mit entsprechend großem folgenden Zeilenabstand hervorgehobenen ersten Zeile (oder mehrerer Zeilen oberhalb des erhaltenen Textes) ist freilich nicht völlig auszuschließen. Es wäre dann aber zu fragen, was vor der Nennung von Bau und Erbauer gestanden haben sollte, um eine derartige Hervorhebung in der ansonsten handwerklich sehr sauber und regelmäßig ausgeführten Inschrift zu verdienen.

\footnotetext{
${ }^{6}$ Ed. Tietz 2003a.
} 
Es ist hingegen als sicher zu betrachten, dass die unterste erhaltene Zeile auch den Abschluss der Inschrift darstellt. Die Gründe hierfür sind sowohl der auch hier sehr breite unbeschriftete Raum sowie das Formular, das den Text mit der Androhung einer Buße für den Missbrauch des Grabes in konventioneller Weise enden lässt.

Z. 1: Mit $\mathfrak{\eta}] \rho \tilde{\omega} v$ liegt wohl ein Steinmetzfehler anstelle von $\mathfrak{\eta}] \rho \tilde{\omega} 0 v$ vor.

Z. 2: Zu erwarten ist hier der Name des Grabherren. Namen auf* ${ }^{\star}$-yndas sind freilich selten. Belegt sind etwa Leoundas in Thesalien, Pholyndas in Epiros ${ }^{8}$ oder Malaundas in Phrygien?. Denkbar wäre aber auch der Name Amyntas. Er ist in Kleinasien sehr beliebt und ist vor allem im nahen Telmessos an prominenter Stelle an der linken Ante des heute nach einer so beannten Person benannten Monumentalgrabs seit dem ausgehenden 4 . Jh. v.Chr. sichtbar ${ }^{10}$, allerdings in leicht abgewandelter Form.

Obwohl der Text auch sonst wohl orthographisch äuffällig ist (Z. 1, korrekte Ergänzung vorausgesetzt), wäre auch die Form Amyndas nicht undenkbar. Ein solcher nämlich ist möglicherweise als Vater eines mutmaßlichen Epheben in einer entsprechenden Liste aus der näheren Umgebung genannt. Sie wurde an dem antiken Platz zwischen Mergenli und Gökbel (wohl das antike Pasanda $)^{11}$ gefunden, der in der Nähe von Kaunos etwa auf halbem Weg zwischen den heutigen Orten Dalaman und Dalyan liegt. Leider ist aber auch dieser Name nur teilweise erhalten. Dieser AMYN $\Delta-^{*}$ kann aus paläographischen Gründen wohl kaum mit dem Urheber der hier vorgestellten Inschrift identisch $\operatorname{sein}^{12}$.

Bei [- - ] YN $\Delta \mathrm{A} \Sigma$ kann es sich im übrigen nicht um eine (irrtümlich geschaffene) Variante des Ethnikons der nahen Polis Kalynda ${ }^{13}$ handeln, das in einer Inschrift aus Kaunos sowie bei bei

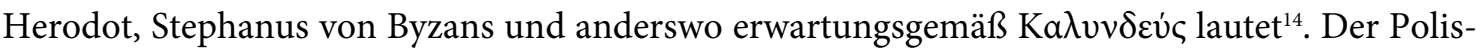
name Kalynda selbst ist ein Neutrum im Plural und kommt daher ebenfalls in keiner korrekten oder durch einfache Verschreibung erklärbaren Form für diese Lücke in Frage ${ }^{15}$. Ein Ethnikon an

\section{${ }^{7}$ IG IX 2517.}

${ }^{8}$ SEG 54, 577.

${ }^{9}$ Drew-Bear 1978, 92 no. 27.

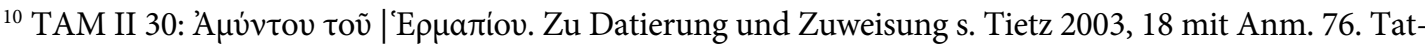
sächlich taucht ein Amyntas, Sohn des Apollonios, Enkel des Apollonios als Vater eines ebenfalls Amyntas genannten Kureten in Ephesos in einer dortigen kaiserzeitlichen Priesterliste auf: I.Ephesos 1037 Z. 6.

${ }^{11}$ Zur Identifikation dieses Platzes mit Pasanda s. Roos 1969, 60; Roos - Herrmann 1971, 31-35; Tietz 2003, 34-35 mit Anm. 156.

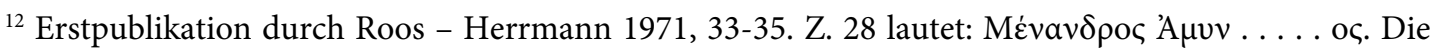
Liste konnte seitdem nicht wieder aufgefunden werden. J. Robert/L. Robert, BE 1972, 465 Nr. 431 merkten an, dass die Namen der Liste gut zum bereits zuvor bekannten Namensbestand der Region passen. Vgl. auch Marek 2006, 234 Nr. 42 (ohne Autopsie), dort "hellenistisch" datiert.

${ }^{13}$ Zur Lokalisierung des Zentralorts von Kalynda in weniger als $15 \mathrm{~km}$ Entfernung von İnlice westlich oberhalb des heutigen Ortes Şerefler s. Roos 1969, 72-73; Tietz 2003, 201-204. Jüngere Argumente gegen diese Lokalisierung wurden jüngst von Onur - Tekoğlu 2020, 10-12 entkräftet.

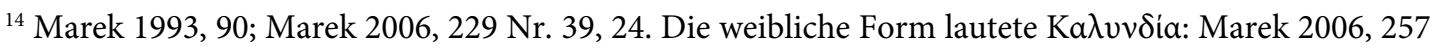
Nr. 73, 7. Hdt. 8, 87-88; daraus St. Byz. s.v. p. 155 (Westermann); Papiri greci e latini Nr. 385. 509. 626.

${ }^{15}$ P. Cair. Zen. 59341 a 8. c 3; Şahin - Adak 2007, 146-151 STR 11-13; Onur - Tekoğlu 2020, 2 R 11. 
dieser Stelle würde außerdem eine ganz unverhältnismäßig lange Textzeile zur Folge haben müssen.

\section{Z 4-5: KAI ГYNAIKI KAI TEKNOI $\Sigma$ AYTOY vgl. TAM II 78, 2}

Z 6: Zu erwarten wäre eingangs der Zeile $[\tau \tilde{\omega} \sigma] v v \tau \rho o ́ \varphi[\omega \varphi$, doch scheint dafür nicht ausreichend Platz zur Verfügung gestanden zu haben. Denkbar wäre eine versehentliche Auslassung des Artikels aufgrund der äußerlich ähnlich endenden Z. 5.

Z. 6: Vgl. TAM II 53 (Telmessos). Der Name Symmasis ist in einer vor kurzem publizierten, aus einer illegalen Grabung stammenden Stiftungsinschrift belegt, die entweder aus Tlos oder Xanthos stammen dürfte ${ }^{16}$. Analog zu den Iotazismen Z. 10 dürfte auch hier $\left.\Sigma\right] v \mu \mu \alpha \sigma[\iota]$ statt $\Sigma] \nu \mu \mu a \sigma[\varepsilon l]$ zu ergänzen sein.

Z. 7: Lücke von 1-3 Buchstaben. Anstelle von $[\Delta$ I?] $\Sigma$ wäre auch ein sehr kurzes Patronym denkbar, das eventuell schon in Z. 6 begonnen haben könnte.

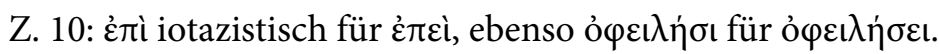

Z. 11: Am Zeilenende Lücke von 2-3 Buchstaben. Da der Text nach dieser Zeile endete, ist eine Angabe zur Höhe der Buße zu erwarten, die ein Grabschänder den Erben des Erbauers leisten sollte. Diese könnte in Drachmen oder Denaren erfolgt sein, abhängig von der Datierung (zur Diskussion des Status der antiken Siedlung bei İnlice siehe unten) vermutlich eher in rhodischen Drachmen.

\section{Überlegungen zur Geschichte Daidalas bis in die Kaiserzeit}

İnlice ist seit langem als antiker Siedlungsplatz bekannt. Auf dem oberhalb des Ortes gelegenen steilen Felsstock von İnlice Asarı befindet sich eine lykische Dynastensiedlung aus der Zeit vor Alexander dem Großen. Sie umfasst eine stark ummauerte Burg, eine ebenfalls ummauerte, auf mehreren Terrassen gelegene Wohnsiedlung, eine recht weiträumige Platzanlage außerhalb der Mauern und, um diese herum sowie sowohl am Siedlungshügel als auch in der gegenüberliegenden Felswand angelegt, eine umfangreiche Nekropole, in der sich auch ein Grabpfeiler befindet ${ }^{17}$.

Auch in der Ebene unterhalb des Burgbergs, wo heute die Überlandstraße D400 verläuft und das Dorf İnlice liegt, sowie am heutigen Ufer des Golfes von Fethiye bei dem Velidada oder - nach seiner heutigen Nutzung - Göcek Halk Plajı genannten Platz wurden bereits zahlreiche antike Überreste entdeckt, darunter ein monumentales Felsgrab vom Tempelfassadentyp unittelbar neben der Straß $\mathrm{e}^{18}$. Eine schlecht erhaltene, doch ursprünglich wohl reich ausgestattete Basilika nahe dem heutigen Ortskern belegt die Siedlungskontinuität zumindest bis in die Spätantike ${ }^{19}$.

${ }^{16}$ Publikation des Textes: Parker 2010. Parker geht ebenda 110 wohl zurecht davon aus, dass der im Text (S. B Z. 7) genannte Symmasis, Sohn des Sortias mit dem Urheber der in der Inschrift umrissenen Stiftung identisch ist (der selbst in den erhaltenen Textteilen lediglich Symmasis genannt wird). Eine Identität mit dem in der hier besprochenen Inschrift Genannten ist also nicht möglich.

${ }^{17}$ Roos 1969, 89. 91-92; Tietz 2003, 232-235; Hellenkemper - Hild 2004, 510; Kolb 2018, 241-242.

18 Tietz 2003, 235-238; Kolb 2018, 242-243.

19 Tietz 2003, 236; Hellenkemper - Hild 2004, 510. 
Seit hellenistischer Zeit hieß der wahrscheinlich Ort Daidala ${ }^{20}$. Er verfügte wohl über ein recht ausgedehntes Territorium einschließlich einiger Inseln $^{21}$ und gelangte noch vor oder sofort $\mathrm{zu}$ Beginn des römischen Krieges gegen Antiochos III. in den festländischen Besitz von Rhodos ('Rhodische Peraia'); es gehörte wohl auch spätestens dann zur 'inkorporierten Peraia' und war so Teil des rhodischen Staatsgebiets, dessen Bewohner das rhodische Bürgerrecht besaßen ${ }^{22}$. Die rhodische Peraia war in der Folge häufig Bedrohungen durch Konkurrenten ausgesetzt, besonders im sogenannten 2. Lykischen Krieg der Rhodier, als Daidala Übergriffen aus dem benachbarten Telmessos ausgesetzt war, das unter pergamenischer Herrschaft stand ${ }^{23}$. Die weitere Geschichte Daidalas, das nie eine eigenständige Polis gewesen zu sein scheint, sondern von den an-


lediglich den Chorographen und einigen wenigen anderen literarischen Quellen weiterhin eine stichwortartige Erwähnung wert ${ }^{25}$.

Immerhin ein wenig Licht scheint durch den hier vorgelegten Text in dieses Dunkel gebracht zu werden können. Bemerkenswert ist nämlich, dass darin jedes Ethnikon oder Demotikon fehlt. Nach einer These von Peter M. Fraser und George E. Bean könnte dies für Inschriften der integrierten, also zum rhodischen Staatsgebiet zählenden Peraia aus dieser Region grundsätzlich typisch sein. Nach diesen erübrigte sich ein Ethnikon auf rhodischem Staatsgebiet, und das angesichts der vielen erhaltenen rhodischen Inschriften auffallend fehlende rhodische Demotikon für Daidala könnte damit erklärbar sein, dass die Bewohner Daidalas und seiner Umgebung Teil eines anderen rhodischen Demos waren ${ }^{26}$. Bei aller Vorsicht angesichts der sehr wenigen erhaltenen Texte aus den umliegenden antiken Orten, kann mit dem hier präsentierten Text die Identifikation des antiken Platzes bei İnlice mit Daidala als Teil der rhodischen Peraia ein wenig plausibler gemacht und aufgrund der wohl kaiserzeitlichen Datierung auf eine spätere Zeit als bisher ausgedehnt werden.

${ }^{20}$ Obwohl bislang nicht eindeutig gesichert ist die Identifikation doch weitgehend unstrittig (Tietz 2003, 231-233; Kolb 2018, 238-239) und ist seit der Lokalisierung von Kalynda bei Kozpınar/Şerefler nur von Şahin - Adak 2007, 152-157 angefochten worden. Dagegen aber zurecht Onur - Tekoğlu 2020, 10-12.

${ }^{21}$ Tietz 2003, 239-243; Kolb 2018, 238-340.

${ }^{22}$ Liv. 37, 22, 3; Strab. 14, 2, 2; Bresson 1999, 107-108. 110; Wiemer 2002, 270; Tietz 2003, 243-244; Hellenkemper - Hild 2004, 510; Kolb 2018, 239-241.

${ }^{23}$ Polyb. 27, 7, 2; Liv. 37, 22, 3; Zimmermann 1993, 117 mit Anm. 40; Wiemer 2002, 267; Tietz 2003,

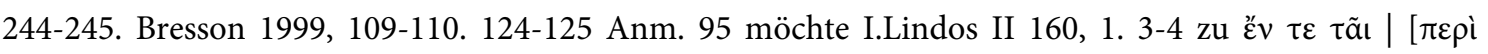

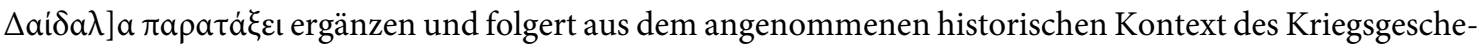
hens um 190 v.Chr. Daidala könne zumindest zu diesem Zeitpunkt nicht Teil der inkorporiertern rhodischen Peraia gewesen sein, da in dieser Inschrift nach ihm belegt ist, der rhodische Hegemon für Lykien sei in Daidala eingeschlossen gewesen, damals die einzige lykische Besitzung der Rhodier. Die vorgeschlagene Ergänzung ist attraktiv, doch die Schlussfolgerungen Bressons sind kaum zwingend.

${ }^{24}$ Strab. 651 ( $\chi \omega$ piov); Ptol. 5, 3, 2 (то́лос); Plin. nat. 5, 103. 131 (oppidum); Hellenkemper - Hild 2004, 510; Kolb 2018, 239-241 mit Anm. 254.

${ }^{25}$ Strab. 651; Plin. nat. 5, 101. 103; Stad. 256-258; Ptol. 5, 3, 3; St. Byz. s.v. Daidala.

${ }^{26}$ Fraser - Bean 1954, 55; vgl. Tietz 2003, 245. 


\section{Das Fehlen Daidalas im Straßenmonument aus Patara}

Im 45 n.Chr. erstellten Verzeichnis der lykischen Überlandstraßen aus Patara taucht Daidala nicht auf ${ }^{27}$. Dies bedeutet nicht, dass die Siedlung zuvor aufgehört hätte zu existieren. Zurecht ist auch schon angemerkt worden, dass eine Zugehörigkeit der Siedlung zur rhodischen Peraia dafür keinen hinreichenden Grund liefert, denn dieses Straßenmonument vermerkt in einem anderen - dem einzigen eindeutigen - Fall die Durchquerung nichtlykischer Territorien sehr wohl ${ }^{28}$. Es scheint somit nahezuliegen, dass Daidala zum Zeitpunkt der Errichtung des Straßenmonuments nicht zu Rhodos gehörte, wenn eine damalige Straße - wie allgemein angenommen - durch sein Territorium führte. Aus diesem Grund hat F. Onur vor kurzem vermutet, dass Daidala in den lykischen Poleis Kalynda oder Telmessos aufgegangen sein könnte, folgend einem Bericht des

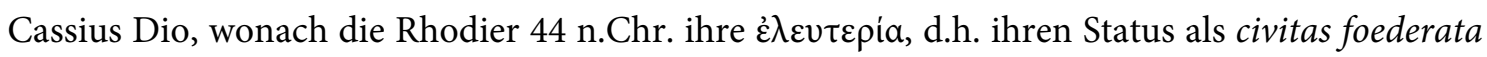
et libera verloren; im Straßenmonument aus Patara sei dann die Nennung einer abhängigen Siedlung an der Strecke kaum als notwendig erachtet worden ${ }^{29}$.

Die Auslassung einer derart großen Siedlung, die unmittelbar an der Strecke lag, erscheint dennoch zumindest ungewöhnlich ${ }^{30}$. Die Verlagerung des Siedlungskerns - vom klassischen Burgberg in die Küstenebene hinunter - bedeutet jedenfalls nicht, dass der Ort nicht von der Straße berührt worden wäre. Im Gegenteil: Die wenigen heute feststellbaren archäologischen Überreste finden sich in der gesamten Schwemmebene bis an die Küste, so dass eine Küstenstraße von Telmessos nach Kalynda den Siedlungskern Daidalas zwangsläufig berühren musste ${ }^{31}$. Eine andere als eine Küstenstraße kommt für den im Straßenmonument genannten Weg nicht in Frage. Die Strecke von Telmessos nach Kalynda wird dort mit 184 Stadien angegeben ${ }^{32}$. Gemeint sind damit wohl attische Stadien zu 185-185,5m, gerechnet aus römischen Meilen, die acht Stadien umfassten ${ }^{33}$. Damit war der Weg zwischen den beiden Orten mit 34,040 bis 34,132 km angegeben.

${ }^{27}$ Die Datierung geht eindeutig aus der Titulatur des Claudius hervor: Marksteiner - Wörrle 2002, 556. Irrig gibt Jones 2001, 161 das Jahr 46 n.Chr. an.

${ }^{28}$ Şahin - Adak 2007, 216 zu STR 42; Onur - Tekoğlu 2020, 12.

${ }^{29}$ Onur - Tekoğlu 2020, 12-13. Cass. Dio 60, 24, 4 als einzige Quelle vermerkt für diesen Vorgang lediglich, Claudius habe den Rhodiern aufgrund von Hinrichtungen römischer Bürger ihre Freiheit genommen.

${ }^{30}$ Mehrere lykische Orte tauchen im Straßenmonument als Durchgangsstationen ohne Abzweigung auf, namentlich Neisa (STR 6-7), Lyrnai (STR 13-14), Hippukome (STR 14-15), Symbra (STR 15-16), Balboura (STR 22-23), Kastabara (STR 27-28), Kodopa (STR 29-30), Akarassos (STR 30-31), Soklai (STR 31-32), Kitanaura (STR 42-43), Typallia (STR 44-45), Onobara (STR 47-48), Kyaneai (STR 56-57), Korykos (STR 64-65) sowie möglicherweise Kandyba (STR 55), und außerdem ein nur fragmentiert erhaltenes Toponym in STR 37-38: Şahin - Adak 2007, passim. Durch die Formulierung ausdrücklich als Durchgangsstation gekennzeichet werden Kollyba (STR 51) sowie bislang nicht identifizierter Ort namens Plata[...] (STR 25). Dass Daidala vermutlich nie den Polisstatus erreichte, dürfte hingegen eine geringere Rolle spielen.

${ }^{31}$ Die gegenteilige Vermutung bei Kolb 2018, 241 ist unzutreffend, und dementsprechend auch seine Hypothese, das Fehlen Daidalas auf dem Straßenmomument sei damit erklärbar. Wichtige Eckpukte hierfür sind die mutmaßlichen Hafenanlagen von Velidada am Meer und die Basilika sowie verschiedentliche weiteren Siedlungsspuren im heutigen Dorf İnlice nördlich der heutigen Landstraße: Tietz 2003, 235-238.

\footnotetext{
${ }^{32}$ Onur - Tekoğlu 2020, 2 R 11.

${ }^{33}$ Şahin - Adak 2007, 120.
} 
Von Ortskern zu Ortskern ${ }^{34}$ beträgt die Entfernung des antiken Telmessos zur Siedlung bei Şerefler/Kozpinar nach heutigem Straßenverlauf $41 \mathrm{~km}$. Diese Straße folgt einer recht direkten Strecke, die lediglich aufgrund schwierigen Geländes stellenweise - besonders unmittelbar östlich von İnlice - vom antiken Weg abweicht, der darauf aufgrund seiner geringeren Breite und der geringeren Geschwindigkeit der dort verkehrenden Fahrzeuge weniger Rücksicht nehmen musste ${ }^{35}$. Für Umwege um den antiken Platz bei İnlice herum ist in der Angabe des Straßenmonuments von Telmessos nach Kalynda jedenfalls sicher kein Raum. Es scheinen im Straßenmonument aber ganz grundsätzlich nur selbständige Städte erwähnt worden zu sein ${ }^{36}$.



Karte 1) Westlykien vom Golf von Fethiye bis Kaunos

(nach Onur - Tekoğlu 2020, 16 Fig. 39, mit freundlicher Genehmigung der Autoren)

\section{Sieben ,fehlende` Kilometer im Straßenmonument aus Patara}

Aber auch, wenn Rhodos durch den Verlust der Freiheit bis zum Widerruf dieses Willkürakts des Claudius durch diesen selbst etwa 53 n.Chr. für einige Jahre Teil der Provinz Asia war ${ }^{37}$ - ein Verlust integrierten Polisterritoriums (einschließlich der dortigen rhodischen Bürger!) muss mit diesem ephemeren Autonomieverlust ${ }^{38}$ keineswegs einhergegangen sein. Das Gegenteil ist vielmehr wahrscheinlich. Rhodos erhielt, nachdem Vespasian es 74 n.Chr. erneut in die Provinz Asia

\footnotetext{
${ }^{34}$ Dass das Straßenmonument so maß, hat Onur 2016, 97-101 dargelegt.

${ }^{35}$ Die antike Küstenstraße war wohl ein wenig kürzer: Şahin - Adak 2007, 109. 146-147 und die anliegende Karte.

36 Tietz 2003, 276. 292; Onur 2016, 105-106.

${ }^{37}$ Schmitt 1957, 189 hebt zurecht hervor, dass die Hinrichtung auch römischen Bürger Teil der Autonomie war, die Rhodos als Inhaberin eines foedus aequm mit Rom de iure fraglos zustand. Zur Restituierung der Libertas s. ebenda; Tac. ann. 12, 58; Suet. Claud. 25; Suet. Nero 7.

${ }^{38}$ Tac. ann. 12, 53 formuliert zur rhodischen libertas, diese sei adempta saepe et firmata und beschreibt damit das wechselhafte Verhältnis Roms zu Rhodos recht gut.
} 
eingegliedert hatte ${ }^{39}$, unter Domitian die libertas und möglicherweise auch Kaunos als Teil der 'unterworfenen' (d.h. nicht integrierten) Peraia zurück ${ }^{40}$, welches es wohl spätestens nach dem Sieg Octavians im römischen Bürgerkrieg 30 v.Chr. verloren hatte ${ }^{41}$. Ein solches 'Geschenk' wäre sicher eher verständlich, wenn es benachbarte, integrierte Teile der rhodischen Peraia gab. Dion von Prusa spricht in seiner Rhodierrede von rhodischen Einkünften aus Karien und Lykien ${ }^{42}$, und dies offensichtlich zu einer Zeit, als beide Regionen bereits provinzialisiert, Rhodos aber eine freie Polis war, und zwar frühestens aber nach dem Tod Neros, der darin erwähnt wird ${ }^{43}$.

Das Straßenmonument aus Patara liefert für diese Vermutung zwei wichtige Indizien. Zum einen sind die dort genannten Strecken - sofern sie sicher genug nachvollziebar sind - in ihrer Länge entweder korrekt (21 von 65$)^{44}$ oder scheinbar zu kurz (4 von 65$)^{45}$ angegeben, keine einzige aber zu lang. Eine davon, STR 26 von Tlos nach Kastabara, ist nach den Untersuchungen von S. Şahin und M. Adak nur so weit angegeben, wie das claudische Straßenprogramm reichte, und nicht, wie es wohl eher der Länge der Straße entsprochen hätte, die etwa $3 \mathrm{~km}$ weiter zwei severische Meilensteine aufweist ${ }^{46}$. Die exakt angegebenen Entfernungen aber machen den überwältigenden Anteil der genannten aus, so dass die 'zu kurzen' Einträge kein Zufall sein dürften und in jedem einzelnen Fall erklärungsbedürftig sind. Zum anderen bleiben auch einige bedeutende lykische Orte in der Inschrift des Straßenmonuments ungenannt, obwohl es zweifellos Verkehrsverbindungen dorthin gab. Dies betrifft etwa das nordwestliche Boubon ebenso wie Antiphellos und andere Poleis an der Küste Zentrallykiens sowie außerdem zahlreiche kleinere antike Orte in ganz Lykien. S. Şahin und M. Adak bemerken dementsprechend zu Boubon, dieses dürfte "während der Einrichtung der Provinz vielleicht außerhalb dieser Neuorganisation" geblieben $\operatorname{sein}^{47}$. Dasselbe dürfte auch für die übrigen fraglichen Orte gelten: Das Straßenmonument lieferte kein

${ }^{39}$ Suet. Vesp. 8, 4; Magie 1950, 569 mit 1387 Anm. 50; Schmitt 1957, 190.

${ }^{40}$ Dion. Prus. or. 31, 125; Magie 1950, 1427 Anm. 9; Schmitt 1957, 190 mit Anm. 6. Marek 2006, 101 102 sieht in Dions Bemerkung, Kaunos sei den Römern wie den Rhodiern untertan, einen Anachronismus und führt als Belege dafür, dass Kaunos zum Zeitpunkt der Rede, und zwar seit der Provinzgründung 43 n.Chr. am ehesten eine Polis innerhalb der Provinz Lykia war, verschiedene Volksbeschlüsse seit den 80er Jahren des 1. Jhs. n.Chr. an (vgl. auch Marek 2011). Angesichts der unsicheren Datierung der Rhodierrede des Dion (dazu unter Abwägung aller Positionen Sidebottom 1992) können dies aber nur punktuelle Hinweise sein, die angesichts der so wechselhaften Geschichte von Rhodos und seinem Territorium kaum hinreichen, um eine derart konkrete zeitgenössische Angabe des Rhetors völlig von der Hand zu weisen. Die Frage muss, besonders angesichts des sehr wechselvollen Verhältnisses der Rhodier zu Rom in der Mitte des 1. Jhs. n.Chr. wohl vorerst offenbleiben.

${ }^{41}$ Schmitt 1957, 187. Köktürk - Milner 2003, 136. Plin. nat. 5, 104 jedenfalls kennt Kaunos als oppidum liberum.

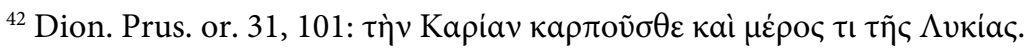

${ }^{43}$ Sidebottom 1992, 408-414. Die Freiheit der Rhodier und die Furcht, diese zu verlieren, ist in Dion. Prus. 31, 112 angesprochen. Der Tod Neros ist in Dion. Prus. 31, 148 vorausgesetzt.

${ }^{44}$ Şahin - Adak 2007, 120-278 STR 1-6. 8-10. 16. 18-20. 22. 28. 60-65.

${ }^{45}$ Şahin - Adak 2007, 120-278 STR 11 (wobei STR 11 die hier zu diskutierende Strecke von Telmessos nach Kalynda ist)-12. 26. 42.

${ }^{46}$ Şahin - Adak 2007, 179.

${ }^{47}$ Şahin - Adak 2007, 165 zu STR 20. 
praktisches Verzeichnis aller wichtigen lykischen Verkehrswege, sondern war als von den Lykiern dediziertes Ehrenmonument ein Mittel der Repräsentation des Claudius und - in gerigerem Maße - seines Statthalter Veranius ${ }^{48}$.

Dies legt den Schluss nahe, dass sich dort lediglich diejenigen Straßen finden, an denen von 43 bis 45 n.Chr. tatsächlich gebaut worden war. Wenn also Daidala 45 n.Chr. noch Teil der integrierten rhodischen Peraia und die Polis Rhodos kurz zuvor beim Kaiser in Ungnade gefallen war, sind das zwei denkbare Gründe, weshalb Daidala im Straßenmonument von Patara fehlt. Die, wie oben angesprochen, an der Strecke von Telmessos nach Kalynda (STR 11) fehlenden ca. 7 km wären also mit der Ost-West-Erstreckung des Territoriums von Daidala gleichzusetzen, das nicht vom Straßenbauprogramm profitierte. Für eine solche Erstreckung des festländischen Territoriums von Daidala sprach bereits vor der Entdeckung des Straßenmonuments einiges: $7 \mathrm{~km}$ entsprechen recht genau der Entfernung zwischen den beiden heutigen Ortschaften Göcek im Westen und Yanıklar im Osten, wenn man nicht der heutigen Überlandstraße folgt, sondern einen direkteren Weg annimmt ${ }^{49}$. Unmittelbar westlich von ersterem erheben sich die steilen Berge, die wohl zu Kalynda gehörten, und Yanıklar liegt in der Ebene des Glaukos, der als Grenzfluss leicht denkbar ist ${ }^{50}$. Diese Vermutung durch die Weise bekräftigt, wie andere Strecken im Straßenmonument behandelt wurden, die nicht durch Gebiet des Lykischen Bundes führten. S. Şahin und M. Adak haben vorgeschlagen, dass bei der Verzeichnung der Strecke von Idebessos nach dem "Kitanaura der Termessier", die mit lediglich $6 \mathrm{~km}$ von ca. $30 \mathrm{~km}$ nur teilweise angegeben sein kann, zum Zeitpunkt der Errichtung des Straßenmonuments eben nur dieses eine Fünftel fertiggestellt gewesen sein könnte, wobei man daran gedacht habe, die restliche Strecke nach Fertigstellung in Farbe auf dem Straßenmonument nachzutragen ${ }^{51}$. Eher ließe sich freilich vermuten, dass das lykische Straßenbauprogramm die übrige Strecke überhaupt nicht betraf, da sie sich im Besitz von Termessos und damit nicht im Zuständigkeitsbereich des Veranius befand. Hier wurden also wohl zwar der Ausgangs- und Zielort einer Straße angeben, aber in Stadien nur diejenigen, die tatsächlich umgesetzt worden waren. Und auch die dritte im Straßenmonument aus Patara deutlich 'zu kurz' angegebene Strecke von Kalynda nach Kaunos (STR 12) dürfte lediglich diejenigen Streckenabschnitte betreffen, welche in der Provinz Lykien erneuert worden waren, denn es ist keine Rede davon, dass - wie im Falle des termessischen Kitanaura - auch Wege außerhalb Lykiens betroffen waren ${ }^{52}$. Spätestens seit dem Fund eines neronischen Zollgesetzes für die Provinz Lykien ist es wahrscheinlich, dass Kaunos bereits bei oder kurz nach der Einrichtung der

\footnotetext{
${ }^{48}$ Marksteiner - Wörrle 2002, 556; Şahin - Adak 2007, 11-15; Onur - Tekoğlu 2020, 12. bei Marksteiner - Wörrle auch die Publikation eines Ehrenmonuments auf dem zentrallykischen Bonda Tepesi, womit die Lykier Claudius und seinen Statthalter Q. Veranius als Wohltäter ehren, unter anderem für den Straßenbau.

${ }^{49}$ Hiervon gehen auch Şahin - Adak 2007 in der anliegenden Karte aus.

${ }^{50}$ Entsprechend ausführliche Überlegungen bei Tietz 2003, 239-241.

${ }^{51}$ Şahin - Adak 2007, 216 zu STR 42.

52 Onur 2016, 103-104. Der Textteil des Straßenmonuments aus Patara, in dem von Tätigkeiten des Veranius außerhalb Lykiens die Rede ist, ist bedauerlicherweise nur sehr lückenhaft erhalten und enthält außer Kibyra und einem Laodikeia (mit unklarer Identifikation) keine Ortsnamen: Şahin - Adak 2007, 290292. Dass Kaunos im Straßenmonument nicht wie Attaleia und Kibyra als Teil einer anderen Provinz kenntlich gemacht wurde, mag wenig bedeuten angesichts des Tatbestands, dass auch bei dem nicht lykischen Termessos kein Zusatz angebracht wurde: Onur 2016, 101-105.
} 
römischen Provinz Lykia aus Asia nach dort wechselte ${ }^{53}$. In welchem Jahr genau und unter welchen Umständen dies geschah und ob es dabei blieb, ist freilich noch unsicher ${ }^{54}$.

Dies ist für die Einschätzung des Fehlens von Daidala im Straßenmonument von großer Bedeutung. Auf solche oder andere Art in der Schwebe dürfte auch die Strecke durch das Gebiet von Daiala gehangen haben, sei es, dass dieses als integraler Teil von Rhodos bewusst nicht vom Straßenbau des Kaisers für Lykien profitierte, sei es, dass die administrative Stellung der Siedlung 45 n.Chr., also nur etwa ein Jahr nach dem Entzug der libertas, noch ungeklärt war und man daher auf eine Nennung des ohne jeden Zweifel an der Strecke von Telmessos nach Kalynda gelegenen Ortes verzichtete. Die an der Entfernungsangabe von Telmessos nach Kalynda fehlenden ca. $7 \mathrm{~km}$ erklären sich jedenfalls wohl am leichtesten dadurch, dass dort aufgrund eines nicht-lykischen Territoriums keine Straßen gebaut wurden.

Eine rhodische Exklave in einer römischen Provinz erscheint auf den ersten Blick nicht recht plausibel. Vor allem angesichts der antiken Berichte über einen Freiheitsverlust der Rhodier wenige Jahre zuvor fällt es schwer zu glauben, Claudius habe auf eine sehr kleine Exklave derselben Rhodier besondere Rücksicht genommen, als er erstmals in Lykien eine römische Provinz einrichtete. Die Geschichte von Rhodos sowie die konkrete kaiserzeitliche Situation in Lykien lassen aber eben dies zumindest als Möglichkeit erscheinen. Die angesprochene Geschichte der Rhodier in ihrem nicht immer einfachen Verhältnis zu Rom zeigt, wie wechselvoll und willkürlich sich die Entwicklung darstellen kann. Physkos (das heutige Marmaris) war mindestens bis ins 2. Jh. n.Chr. eine rhodische Exklave in der römischen Provinz Asia ${ }^{55}$. Im übrigen ist integrierter rhodischer Besitz außerhalb der Insel selbst, der durch Strategen verwaltet wurde, bis in severische Zeit belegt ${ }^{56}$. Im Straßenmonument von Patara selbst scheint eine Exklave von Termessos angedeutet zu sein. Es ist jedenfalls keineswegs gesichert, dass die 'Kitanaura der Termessier' genannte Siedlung eine territoriale Verbindung mit dem Kerngebiet von Termessos besaß; ebensogut könnte es sich um eine termessische Exklave in der Provinz Lykien handeln ${ }^{57}$.

\section{Neues Licht auf alte Quellen: Das Zeugnis der Inschrift SEG 53-1440 (ed. Köktürk - Milner 2003)}

Aus einer Privatsammlung in Fethiye wurde im Jahr 2003 eine Inschrift publiziert ${ }^{58}$, die nahelegt, dass es noch lange nach der Umwandlung Lykiens in eine römische Provinz eine gemeinsame Landgrenze von Rhodos und den lykischen Bewohnern dieser Provinz gab. Der Text schildert den wohl von einer Frau (vielleicht namens Artemikleia) angestoßenen Rechtsstreit um den illegalen Verkauf dieses gepachteten und weiterverpachteten öffentlichen Landes an Fremde ( $\xi \dot{\varepsilon} v o \iota)$. Der 'Verkauf durch die Pächter war teils offen abgewickelt worden, teils über Bürger, die als Strohmänner dienten. In beiden Fällen, so der Text, sei das Land schließlich in der Hand von Lykiern gewesen, wobei einige Pächter angeblich den Pachtzins weiterzahlten, um den illegalen

\footnotetext{
${ }^{53}$ Marek 2011, 58-59.

${ }^{54}$ Onur 2016, 105 verweist wohl zurecht auf Dion. Prus. 31, 50. 124-125 (frühestens um 70 n.Chr. zu datieren), worin der Verlust der Freiheit durch die Kaunier angesprochen wird.

${ }^{55}$ Fraser - Bean 1954, 85; Köktürk - Milner 2003, 136.

${ }^{56}$ Schmitt 1957, 191f. mit Anm. 3.

${ }^{57}$ Onur 2016, 101-103.

${ }^{58}$ Köktürk - Milner 2003. Der Inschriftenträger wurde nach Angaben des Eigentümers aus einem Haus „near Fethiye“ nach dort verbracht.
} 
Verkauf zu verschleiern. Die Söhne und Erben zweier Verkäufer standen nun vor Gericht, verklagt auf Wiedergutmachung. Sie gaben jedoch an, ihre Väter hätten das Land rechtmäßig gekauft und dann weiterverkauft. Als Grund für den Verkauf wird angegeben, dass die Grundstücke zu

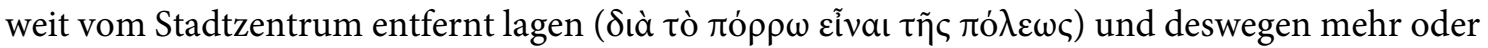
weniger zwangsläufig vernachlässigt gewesen seien. Zumindest einer der Erben wurde dennoch verurteilt, weil er sowohl die Frau als auch die Polis geschädigt habe.

Die genannten Nichtbürger, die als Landkäufer auftraten, dürften identisch mit den Lykiern sein, in deren Hand sich das Land später befand ${ }^{59}$. Dass zunächst nur von Fremden gesprochen wird, hat seinen Grund im juristischen Tatbestand des Verbots des Verkaufs von Land an Nicht-Bürger $^{60}$. Aus diesem Grund lag nicht nur eine Schädigung einer gewissen Artemikleias (entweder die zuvor genannte 'Frau' selbst oder deren Erbin) vor, sondern auch der Polis, die einen Teil ihres Territoriums verloren hatte.

Die exakte Herkunft dieses Textes bzw. der ursprüngliche Aufstellungsort sind unbekannt. Allzu weit dürfte er freilich nicht gereist sein, denn es handelt sich bei dem Textträger um das Fragment einer Stele aus lokalem, um Fethiye herum anstehendem Kalkstein ${ }^{61}$. Es liegt ferner nahe, dass der widerrechtliche Verkauf sich in einem Gebiet abspielte, wo Lykien an das Territorium einer anderen Polis grenzte. In dieser anderen Polis spielte sich der Rechtsstreit ab, und auf deren Territorium muss anschließend die Stele mit dieser Inschrift errichtet worden sein, möglicherweise auf dem in Frage stehenden Land selbst ${ }^{62}$. Wie auch in der Inschrift selbst von einem Prozessbeteiligten angegeben, dürfte sich dieses Land sehr weit vom Poliszentrum entfernt befunden haben, denn die Vergabe öffentlichen Landes zur Pacht an eigene Bürger anstelle einer dauerhaften Vergabe war in der Kaiserzeit ein weitverbreitetes Mittel, um dessen Bewirtschaftung sicherzustellen $^{63}$.

Für die Frage der Grenznachbarn Lykiens und damit der infrage kommenden Poleis ist die Datierung des Textes von großer Bedeutung. Paläographisch wurde er von den Herausgebern in die Zeit zwischen dem späten 1. und dem mittleren 2. Jh. n.Chr. datiert ${ }^{64}$. Weitere Hinweise lieferte die Onomastik der Inschrift, die vor allem in den Ägäisraum und nach Rhodos deutet; einer der Angeklagten, (Claudius) Mnasagoras, und dessen Vater Claudius Antipatros können prosopographisch mit sehr wohlhabenden Mitgliedern einer vornehmen Familie aus Lindos um 100

\footnotetext{
${ }^{59}$ Köktürk - Milner 2003, 143.

${ }^{60}$ Ferrary - Rousset 1998, 301. 309. 313. 323; Köktürk - Milner 2003, 135 mit einigen Belegen aus Delphi und der rhodischen Peraia, vor allem aber mit dem Hinweis, dass das Recht, Land in einer Polis zu erwerben ('̌ $\gamma \kappa \tau \eta \sigma \iota \varsigma)$, an Fremde als besondere Ehre verliehen zu werden pflegte und ansonsten den eigenen Bürgern vorbehalten war. Zur großen Seltenheit solcher Fälle im rhodischen Staat s. Boyxen 2018, 303-304. Robert 1937, 382 nennt zwar etliche hellenistische und kaiserzeitliche ,Fremde' als Eigentümer auswärtiger lykischer Grundstücke, doch handelt es sich um Bürger anderer lykischer Poleis, die hierfür nicht als Parallele taugen.
}

${ }^{61}$ Köktürk - Milner 2003, 131.

${ }^{62}$ So Köktürk - Milner 2003, 134.

${ }^{63}$ Ferrary - Rousset 1998, 336-342; Köktürk - Milner 2003, 135.

${ }^{64}$ Köktürk - Milner 2003, 131-132. 
n.Chr. identifiziert werden ${ }^{65}$. Dass es sich bei der im Text angesprochenen Polis um Rhodos handelt, scheint also sicher, und dass Rhodos zum Zeitpunkt des Rechtsstreits dürfte Rhodos frei war, da ansonsten wohl die Römer einbezogen worden wären ${ }^{66}$. Während die Herausgeber der Inschrift eine Datierung des Rechtsstreits in die erste Hälfte des 2. Jhs. v.Chr. legen und damit implizit an die durch Domitian eingeleitete Phase der rhodischen Freiheit denken ${ }^{67}$, wäre auch ein Ansatz des Landverkaufs in den Jahren zwischen der Restitution der Freiheit durch Claudius (angeblich auf Fürsprache des jungen Nero) und deren erneute Rücknahme durch Vespasian (53-74 n.Chr.) denkbar.

Rhodische Besitzungen von landwirtschaftlichem Interesse, die an Lykien angrenzten, lagen selbstverständlich sämtlich weit genug von der Insel Rhodos entfernt, um die oben erwähnte Begründung für eine Vernachlässigung des Landes plausibel erscheinen zu lassen. Im fraglichen Zeitraum handelt es sich um Kaunos im Westen, die zweifellos zur inkorporierten Peraia gehörige, nach Plinius dem Älteren aber verlassene Insel Megiste vor der lykischen Südküste ${ }^{68}$ und möglicherweise um Daidala, das zwischen Kaunos und Telmessos lag. An Verpachtungen solchen öffentlichen Landes dürfte Dion von Prusa in seiner Rhodierrede gedacht haben, als er von Einkünften aus Lykien und Karien sprach ${ }^{69}$. Kaunos grenzte zweifellos an lykisches Gebiet. Es war freilich nie vollständig in den rhodischen Staat inkorporiert, und so ist die Existenz öffentlichen rhodischen Landes auf dem Territorium von Kaunos zwar denkbar ${ }^{70}$, aber nicht in großem Umfang wahrscheinlich. Kaunos war spätestens 51/50 v.Chr., wahrscheinlich aber schon 67 v.Chr. Teil der Provinz Asia geworden und in der Folgezeit wohl immer Teil einer römischen Provinz ${ }^{71}$.

Der nach Plinius desolate Zustand der Besiedlung auf Megiste wiederum lässt zunächst durchaus an eine Situation wie die in der Inschrift geschilderte denken, in der rhodische Grundeigentümer ihr Land nicht mehr bewirtschaftet haben und der rhodische Staat keine Pächter mehr gefunden haben könnten. Gegen Megiste als Herkunftsort der Inschrift, die durchaus per Schiff in den Raum um Fethyie gelangt sein könnte, spricht aber einiges. Erstens fehlt jeder Hinweis auf eine lykische Übernahme der Insel, zweitens gibt es auf Megiste keine Ackerflächen, die groß genug gewesen wären, um den Aufwand eines Rechtsstreits in den höchsten Kreisen der rhodischen Oberschicht auszulösen, und drittens erwähnt auch die Inschrift mit keinem Wort, dass es sich bei den Ländereien um solche auf einer kleinen Insel handelte ${ }^{72}$.

Aufgrund dessen, vorrangig aber aufgrund des Fundortes der Inschrift haben sich alle bisherigen Kommentatoren, wenn auch vorsichtig, für das Gebiet um Daidala als denjenigen Teil der

\footnotetext{
${ }^{65}$ Köktürk - Milner 2003, 135. Boyxen 2018, 305.

${ }^{66}$ Köktürk - Milner 2003, 136.

${ }^{67}$ Köktürk - Milner 2003, 136-137; vgl. SEG 53-1707 (ca. 100-142 AD).

${ }^{68}$ Plin. nat. 5, 131: Megista, cuius civitas interiit. Die Zugehörigkeit zum rhodischen Staat steht außer Frage: Bresson 1999, 104-106. Die neben ihrer vorrangig militärischen Bedeutung auch landwirtschaftliche Nutzung Megistes belegen meherer Dutzend Gehöfte und einige Pressanlagen: Boyxen 2018, 301 mit Anm. 9.

${ }^{69}$ Dion. Prus. 31, 101.

${ }^{70}$ Die Liste solcher Grundstücke in Lykien bei Robert 1937, 382 setzt sich aus individuellen Schenkungen zusammen.

${ }^{71}$ Marek 2006, 100-102; Marek 2011.

${ }^{72}$ So Köktürk - Milner 2003, 137.
} 
rhodischen Peraia ausgesprochen, der in diesem Prozess zur Rede stand ${ }^{73}$. Vor allem aber spricht die Abfassung des Textes in der ionisch-attischen Koine anderer kaiserzeitlicher Inschriften aus Lykien oder auch aus Kaunos (anstelle des rhodischen Dorisch) ${ }^{74}$ gegen die Aufstellung auf einer vornehmlich von Rhodiern besiedelten Insel oder einer anderen Region, wo der dorische Dialekt vorherrschte. Sie dürfte mithin in einer Region aufgestellt gewesen sein, wo die Mehrheit der Bevölkerung keine Rhodier waren, sondern Lykier auf dem Festland. Daidala ist also dasjenige rhodische Territorium, wo der geschilderte Rechtsstreit seinen Ausgang genommen haben dürfte. Damit wäre seine Zugehörigkeit zu Rhodos zumindest bis in flavische Zeit gesichert.

\section{Schluss}

Die Umgebung von Daidala/İnlice dürfte also vor, nach und auch während der unruhigen Zeit der Provinzialisierung Lykiens unter Quintus Veranius, die 43 n.Chr. ihren Anfang nahm und mit dem Straßenmonument von Patara 45 n.Chr. einen ihrer sichtbarsten Abschlüsse erhielt, integraler Teil der Polis Rhodos gewesen sein, unabhängig davon, ob diese ihre libertas vollständig verloren oder noch letzte Reste einer scheinbaren Selbstverwaltung ${ }^{75}$ bewahrt hatte. So löst sich auch eine weitere bislang offene Frage, nämlich die, weshalb ein offensichtlich so ausgedehnter antiker Platz wie Daidala im Straßenmonument von Patara nicht genannt wurde. An der Straße von Telmessos nach Kalynda lag dieser ja zweifellos. Erklärt man das Schweigen der Inschrift mit der - aus welchen Gründen auch immer - ausgebliebenen Bautätigkeit der römischen Verwaltung, so muss selbiges jedenfalls nicht bedeuten, dass der Ort klein und von geringer Bedeutung war.

Die hier vorgelegte Inschrift, die zweifelsfrei aus dem heutigen Dorf İnlice in der Schwemmebene am Fuß des Burgbergs von İnlice Asarı stammt, ist jedenfalls in Verbindung mit den übrigen in den letzten 30 Jahren ans Licht gekommenen Befunden ein weiterer Hinweis auf die Kontinuität eines Siedlungsplatzes in der Ebene von İnlice. Die heute sichtbaren Siedlungsreste sind wenig aufsehenerregend, doch mit dem anzunehmenden Grabmonument, von dem die Inschrift stammen dürfte, ist immerhin ein weiterer bedeutender Befund hinzugekommen. Es sei auch daran erinnert, dass die bislang dokumentierten Reste ein recht großes Areal abdecken, was umso bemerkenswerter ist, da die Siedlung in der Ebene größtenteils unter jahrhundertaltem Schwemmland begraben liegt, das noch nie eingehend untersucht wurde. Systematische archäologische Untersuchungen im gesamten Gebiet der Fundstreuung, die es an diesem Platz noch nie gegeben hat, wären sicher vielversprechend.

\section{Bibliographie}

Boyxen 2018

B. Boyxen, Fremde in der hellenistischen Polis Rhodos, Berlin 2018.

Bresson 1999

A. Bresson, Rhodes and Lycia in Hellenistic Times, in: V. Gabrielsen (Hg.), Hellenistic Rhodes. Politics, Culture, and Society, Aarhus 1999, 98-131.

\footnotetext{
${ }^{73}$ Boyxen 2018, 304.

${ }^{74}$ Köktürk - Milner 2003, 137. In der kaunischen Epigraphik kommen lediglich einzelne Dorismen als kunstsprachliche Elemente vor: Marek 2006, 117.

${ }^{75}$ Von echter Autonomie lässt sich schon seit dem 2. Jh. v.Chr. nicht mehr sprechen, als Rhodos zum Klientelstaat Roms geworden war, das es nach Belieben dirigierte oder bei Bedarf unterwarf: Bresson 1999, 106-111; Wiemer 2002, 317-328.
} 
Drew-Bear 1978

Ferrary - Rousset 1998

Fraser - Bean 1954

Hellenkemper - Hild 2004

Jones 2001

Köktürk - Milner 2003

Kolb 2018

Magie 1950

Marek 1993

Marek 2006

Marek 2011

Marksteiner - Wörrle 2002

Onur - Tekoğlu 2020

Onur 2016

Parker 2010

Robert 1937

Roos - Herrmann 1971

Roos 1969

Şahin - Adak 2007

Schmitt 1957

Sidebottom 1992
Th. Drew-Bear, Nouvelles Inscriptions de Phrygie, Zutphen 1978.

J.-L. Ferrary - J. Rousset, Un lotissement de terres à Delphes au II ${ }^{\mathrm{e}}$ siècle après J.-C., BCH 122, 1998, 277-342.

P. M. Fraser - G. E. Bean, The Rhodian Peraea and Islands, Oxford 1954.

H. Hellenkemper - F. Hild, Lykien und Pamphylien, TIB 8, Wien 2004.

C. P. Jones, The Claudian Monument at Patara, ZPE 137, 2001, 161168.

H. Köktürk - N. P. Milner, A land Dispute from the Lycian Borderland, AnatSt 53, 2003, 131-138.

F. Kolb, Lykien. Geschichte einer antiken Landschaft, Darmstadt 2018.

D. Magie, Roman Rule in Asia Minor I. II, Princeton 1950.

Chr. Marek, 1992 Çankırı ve Kastamonu'da Araştırmalar Kaunos ve Patara Kazılarında Epigrafik Araştırma, AST XI, 1993, 85-104.

Chr. Marek, Die Inschriften von Kaunos, Vestigia 55, München 2006.

Chr. Marek, Kaunos und Lykien, Adalya 14, 2011, 57-62.

T. Marksteiner - M. Wörrle, Ein Altar für Kaiser Claudius auf dem Bonda Tepesi zwischen Myra und Limyra, Chiron 32, 2002, 545569.

F. Onur - Ş. R. Tekoğlu, The Ancient Routes and New Lycian Inscriptions around Fethiye, Gephyra 19, 2020, 1-32.

F. Onur, Parerga to the Stadiasmus Patarensis (16) The Roads, Settlements and Territories, Gephyra 13, 2016, 89-118.

R. Parker, A funerary foundation from Hellenistic Lycia, Chiron 40, 2010, 103-121.

L. Robert, Études Anatoliennes, Paris 1937.

P. Roos - P. Herrmann, Zusätzliches zum südöstlichen Karien, OpAth 10, 1971, 31-40.

P. Roos, Topographical and other Notes on South-Eastern Caria, OpAth 9, 1969, 59-93.

S. Şahin - M. Adak, Stadiasmus Patarensis. Itinera Romana Provinciae Lyciae [Gephyra Monogr. 1], Istanbul 2007.

H. H. Schmitt, Rom und Rhodods. Geschichte ihrer politischen Beziehungen seit der ersten Berührung bis zum Aufgehen des Inselstaates im römischen Weltreich, München 1957.

H. Sidebottom, The Date of Dio of Prusa's Rhodian and Alexandrian orations, Historia 41, 1992, 407-419. 
Tietz 2003

Tietz 2003a

Wiemer 2002

Zimmermann 1993

W. Tietz, Der Golf von Fethiye. Politische, ethnische und kulturelle Strukturen einer Grenzregion vom Beginn der nachweisbaren Besiedlung bis in die römische Kaiserzeit [Antiquitas 1.50], Bonn 2003. W. Tietz, Lissai und Kaunos. Eine neue Grabinschrift aus dem lykisch-karischen Grenzgebiet, EA 36, 2003, 121-126.

H.-U. Wiemer, Krieg, Handel und Piraterie. Untersuchungen zur Geschichte des hellenistischen Rhodos [Klio Beih. 6], Berlin 2002.

M. Zimmermann, Bemerkungen zur rhodischen Vorherrschaft in Lykien (189/88-167 v.Chr), Klio 75, 1993, 110-130.

\section{İmparatorluk Dönemi'nde Batı Lykia'da Daidala: \\ Yeni Bir Yazıt ve Tarihi Gözlemler \\ Özet}

Bu makalede sunulan yazıt şüphesiz İnlice (Muğla/Fethiye) arkeoloji alanında bulunmuş olan ilk yazıttır. Geç Helenistik Dönem ya da Erken İmparatorluk Çağı'na ait olan yazıt, bulunduğu yerin Rhodos Peraiası'na ait olduğuna ve Peraia'nın kurulduğu döneme işaret etmektedir. Daidala'nın İ.S. 46 yılında Patara'da dikilen Stadiasmus'ta anılmaması, yerleşimin Rhodos Devleti toprakları içerisinde kalması ve bu nedenle de yol programına dahil edilmemiş olması ile açıklanmaktadır.

Yazıtın çevirisi şöyledir: “Bu mezarı (“heroon”), Apollonios oğlu Apollonios'un izniyle Amyndas kendisi ve eşi için yaptırdı. Ben (Amyndas), ayrıca süt kardeşim Symmasis oğlu Symmasis ve ondan olan çocuklara izin veriyorum. Başka hiç kimseye defnetme izni yoktur, aksi takdirde varislerime [... miktarında ceza ödeyecektir]."

Anahtar Sözcükler: Daidala, Lykia, Rhodos, Peraia, Stadiasmus Patarensis.

Daidala in Western Lycia in the Imperial Age:

A New Inscription and Some Historical Considerations

Abstract

In this article, the first inscription, which without any doubt was originally found at the archaeological site of İnlice (Muğla/Fethiye), is presented. The text from late Hellenistic/early imperial times hints at the place belonging to the Rhodian Peraia at the time of its creation. The fact that Daidala is left out in the Stadiasmus from Patara from AD 45 is explained by Daidala belonging to the state of Rhodes and thus not included in the imperial building program.

Keywords: Daidala, Lycia, Rhodos, Peraia, Stadiasmus from Patara. 